\title{
Inversion Formula for a Radon-Type Transform Arising in Photoacoustic Tomography with Circular Integrating Detectors
}

\author{
Sunghwan Moon \\ Department of Mathematics, College of Natural Sciences, Kyungpook National University, Daegu 41566, Republic of Korea \\ Correspondence should be addressed to Sunghwan Moon; sunghwan.moon@knu.ac.kr
}

Received 21 March 2018; Revised 5 May 2018; Accepted 4 June 2018; Published 3 July 2018

Academic Editor: Antonio Scarfone

Copyright (C) 2018 Sunghwan Moon. This is an open access article distributed under the Creative Commons Attribution License, which permits unrestricted use, distribution, and reproduction in any medium, provided the original work is properly cited.

\begin{abstract}
This paper is devoted to a Radon-type transform arising in a version of Photoacoustic Tomography that uses integrating circular detectors. The Radon-type transform that arises can be decomposed into the known Radon-type transforms: the spherical Radon transform and the sectional Radon transform. An inversion formula is obtained by combining existing inversion formulas for the above two Radon-type transforms.
\end{abstract}

\section{Introduction}

Hybrid biomedical imaging is an emerging technology using the combination of different physical signals in order to utilize their advantages to enhance image. Among hybrid biomedical imaging, thermoacoustic tomography (TAT) and its sibling Photoacoustic Tomography (PAT) are the most successful examples. Many computed tomography researchers have been considering the mathematical modeling of PAT/TAT and many breakthroughs have been achieved. Also, mathematical problems arising in PAT/TAT also relate to sonar and radar research (e.g., $[1,2])$.

TAT/PAT utilizes ultrasound and optical or radiofrequency electromagnetic waves. While pure ultrasound imaging typically offers high resolution images with low contrast, optical or radiofrequency electromagnetic imaging offers an enormous contrast between cancerous and healthy tissues and low resolution. After the photoacoustic effect discovered by A.G. Bell [3], one can take advantage of the strengths of the pure optical and ultrasound imaging and form the basis of TAT/PAT.

In TAT, a very short radiofrequency (RF) pulse is sent through a biological object, more or less uniformly irradiated. RF energy, absorbed throughout the object, causes thermoelastic expansion of the tissue and emergence and propagation of a pressure wave (an ultrasound signal). This pressure wave can be measured by transducers placed outside the object. The spatial distribution of the absorption of RF energy will have a very good contrast. This is due to the fact that cancerous cells absorb several times more energy than healthy tissue in the RF range. Since the intensity of the source of the acoustic wave describes the spatial distribution of the absorption of RF energy, one of the classical mathematics problems of TAT is the recovery of this initial acoustic pressure field from the ultrasound measurements made outside the object.

PAT differs from TAT only in the way the thermoacoustic signal is triggered. PAT uses a laser pulse rather than an RF. The rest of the procedure is the same as that of TAT. From the mathematical view, there is no difference between TAT and PAT [4]. Hence, from now on, we will just mention PAT.

As the classic detector, small piezoelectric transducers were used in PAT. Since these transducers mimic point-like measurements, reconstruction algorithms produce images with a spatial resolution limited by the size of the transducers. Another drawback of such detectors is the difficulty in manufacturing small transducers with high sensitivity. To overcome these weak points, various other types of acoustic detectors have been introduced, e.g., linear, planar, and cylindrical detectors [5-9]. These detectors are modeled as measuring the integrals of the pressure over the shape of the detector. Linear, planar, and cylindrical detectors do not provide a simple and compact experimental buildup (because their ideal size is infinite). 
Zangerl et al. started to consider PAT with circular integrating detectors [10-12] (which can be realized with line-profile transducer) [13]. Some works [11, 12, 14] have considered PAT with circular integrating detectors on cylindrical geometry: a stack of parallel circular detectors whose centers lie on a cylinder. The cases where the centers of the detector circles are located on a plane or a sphere have been discussed in [14]. In [10-12] they showed that the data from PAT with circular detectors was a solution of a certain initial value problem and converted this problem into a circular Radon transform using this fact. In [10] the spherical geometry, which can be useful in investigating small object, was discussed. Here we also study PAT with the circular integrating detectors on a spherical geometry which is already discussed in [10]. Our approach is to define a new Radon-type transform arising in this version of PAT, and we show that this transform can be decomposed into the spherical Radon transform and the sectional Radon transform introduced by Rubin $[15,16]$, both of which are already studied.

This paper is organized as follows. Section 2 is devoted to the construction of a Radon-type transform arising in PAT with circular detectors. In Section 3, we show that the Radontype transform is the composition of the sectional Radon transform and the spherical Radon transform with centers on the boundary of a sphere, and we present the inversion formula using this fact.

\section{Photoacoustic Tomography with Circular Integrating Detectors}

In PAT, the acoustic pressure $p(\mathbf{x}, t)$ satisfies the following initial value problem:

$$
\begin{aligned}
& \partial_{t}^{2} p(\mathbf{x}, t)=\Delta_{\mathbf{x}} p(\mathbf{x}, t) \\
& \qquad(\mathbf{x}, t)=\left(x_{1}, x_{2}, x_{3}, t\right) \in \mathbb{R}^{3} \times(0, \infty), \\
& p(\mathbf{x}, 0)=f(\mathbf{x}) \quad \mathbf{x} \in \mathbb{R}^{3}, \\
& \partial_{t} p(\mathbf{x}, 0)=0 \quad \mathbf{x} \in \mathbb{R}^{3} .
\end{aligned}
$$

(We assume that the sound speed everywhere including the interior of the object is equal to one.) The goal of PAT is to recover the initial pressure $f$ from measurements of $p$ outside the support of $f$.

Throughout this article, it is assumed that the initial pressure field $f$ is smooth and supported in the unit ball $B^{3}$. We also assume that the acoustic signals are measured by a set of circular detectors on the sphere in the plane perpendicular to $(\boldsymbol{\theta}, 0) \in S^{2}$ with distance $s \in[-1,1]$ from the origin, where $\boldsymbol{\theta} \in S^{1}$ (see Figure 1).

Basically, the spherical geometry could be very useful for the investigation of small objects. Here we studied the spherical geometry Zangerl et al. suggested [10].

The measured data $P(\boldsymbol{\theta}, s, t)$ for $(\boldsymbol{\theta}, s, t) \in S^{1} \times[-1,1] \times$ $[0, \infty)$ can be written as

$$
P(\boldsymbol{\theta}, s, t)=\frac{1}{2 \pi} \int_{\overline{\boldsymbol{\theta}} \cdot \boldsymbol{\alpha}=s, \boldsymbol{\alpha} \in S^{2}} p(\boldsymbol{\alpha}, t) \mathrm{d} S(\boldsymbol{\alpha}),
$$

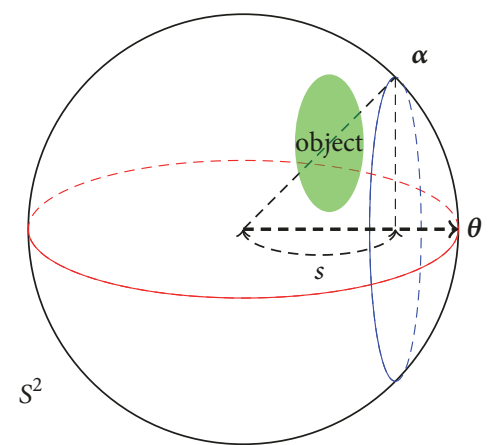

FIgURE 1: The circle on the sphere in the plane perpendicular to $(\boldsymbol{\theta}, 0) \in S^{2}$ with distance $s$ from the origin.

where $\overline{\boldsymbol{\theta}}=(\boldsymbol{\theta}, 0) \in S^{2}$ and $\mathrm{d} S$ (everywhere in the text $\mathrm{d} S$ denotes the measure either on the circle or on the sphere; the dimension will be clear from the context) is the measure on the unit circle $S^{1}$. The shape of detectors is the circle which lies in a plane perpendicular to $\overline{\boldsymbol{\theta}}$ with distance $s$ from the origin (see Figure 1). Also, it is a well-known fact that

$$
p(\mathbf{x}, t)=\partial_{t}\left(\frac{1}{4 \pi t} \int_{\partial B^{3}(\mathbf{x}, t)} f(\boldsymbol{\beta}) \mathrm{d} S(\boldsymbol{\beta})\right)
$$

is a solution of $\operatorname{PDE}(1)$ (see [17]). Here $B_{t}^{3}(\mathbf{x})=B^{3}(\mathbf{x}, t)$ is a ball in $\mathbb{R}^{3}$ centered at $\mathbf{x} \in \mathbb{R}^{3}$ with radius $t$ and $\mathrm{d} S$ is the standard measure on the sphere. Hence $P(\boldsymbol{\theta}, s, t)$ becomes

$$
\begin{aligned}
& P(\boldsymbol{\theta}, s, t) \\
& =\frac{1}{2 \pi} \int_{\overline{\boldsymbol{\theta}} \cdot \boldsymbol{\alpha}=s, \boldsymbol{\alpha} \in S^{2}} \partial_{t}\left(\frac{1}{4 \pi t} \int_{\partial B^{3}(\boldsymbol{\alpha}, t)} f(\boldsymbol{\beta}) \mathrm{d} S(\boldsymbol{\beta})\right) \mathrm{d} S(\boldsymbol{\alpha}) \\
& =\frac{1}{8 \pi^{2}} \partial_{t}\left(t \int_{\overline{\boldsymbol{\theta}} \cdot \boldsymbol{\alpha}=s, \boldsymbol{\alpha} \in S^{2}} \int_{S^{2}} f(\boldsymbol{\alpha}+t \boldsymbol{\beta}) \mathrm{d} S(\boldsymbol{\beta}) \mathrm{d} S(\boldsymbol{\alpha})\right) .
\end{aligned}
$$

Let us define, for $(\boldsymbol{\theta}, s, t) \in S^{1} \times[-1,1] \times[0, \infty)$,

$$
\mathscr{R}_{P} f(\boldsymbol{\theta}, s, t)=\int_{\bar{\theta} \cdot \boldsymbol{\alpha}=s, \boldsymbol{\alpha} \in S^{2}} \int_{S^{2}} f(\boldsymbol{\alpha}+t \boldsymbol{\beta}) \mathrm{d} S(\boldsymbol{\beta}) \mathrm{d} S(\boldsymbol{\alpha}) .
$$

Then, (4) reads as

$$
P(\boldsymbol{\theta}, s, t)=\frac{1}{8 \pi^{2}} \partial_{t}\left(t \mathscr{R}_{P} f(\boldsymbol{\theta}, s, t)\right) .
$$

If $f$ is odd in $x_{3}$, i.e., $f\left(x_{1}, x_{2}, x_{3}\right)=-f\left(x_{1}, x_{2},-x_{3}\right)$, then $\mathscr{R}_{P} f$ is equal to zero. We thus assume that $f$ is even in $x_{3}$, i.e., $f\left(x_{1}, x_{2}, x_{3}\right)=f\left(x_{1}, x_{2},-x_{3}\right)$. Indeed, any function compactly supported in the upper hemisphere of $B^{3}$ can be extended to an even function with support in $B^{3}$. This means that, for any object located on only the hemisphere of $B^{3}$, we can reconstruct its image through even extension. We will demonstrate that $\mathscr{R}_{P}$ is the composition of the spherical Radon transform centered at $S^{2}$ and the sectional Radon transform. This will allow us to recover $f$ from $P$. 


\section{Reconstruction}

We first show that $\mathscr{R}_{P}$ can be decomposed into the spherical Radon transform centered at $S^{2}$ and the sectional Radon transform.

Definition 1.

(1) The sectional Radon transform $Q$ maps a function $\phi$ on $S^{1} \times[-1,1] \times[0, \infty)$ with $\phi\left(\alpha_{1}, \alpha_{2}, \alpha_{3}, t\right)=$ $\phi\left(\alpha_{1}, \alpha_{2},-\alpha_{3}, t\right)$ into

$Q \phi(\boldsymbol{\theta}, s, t)=\int_{\overline{\boldsymbol{\theta}} \cdot \boldsymbol{\alpha}=s} \phi(\boldsymbol{\alpha}, t) \mathrm{d} S(\boldsymbol{\alpha})$,

$$
\text { for }(\boldsymbol{\theta}, s, t) \in S^{1} \times[-1,1] \times[0, \infty) \text {. }
$$

(2) The spherical Radon transform $R_{S}$ maps a locally integrable function $f$ on $\mathbb{R}^{3}$ into

$$
R_{S} f(\alpha, t)=\int_{S^{2}} f(\boldsymbol{\alpha}+t \boldsymbol{\beta}) \mathrm{d} S(\boldsymbol{\beta}),
$$

$$
\text { for }(\boldsymbol{\alpha}, t) \in S^{2} \times[0, \infty) \text {. }
$$

Now it is easily seen that the following representation of $\mathscr{R}_{P} f$ holds.

Theorem 2. For any $f \in C_{c}^{\infty}\left(\mathbb{R}^{3}\right)$, we have $\mathscr{R}_{P} f(\boldsymbol{\theta}, s, t)=$ $Q\left(R_{S} f\right)(\boldsymbol{\theta}, s, t)$.

The spherical Radon transform is a classic object in computed tomography. Many inversion formulas for this transform have been derived in [18-20]. The sectional Radon transform was introduced in $[15,16,21,22]$ and the relation between the sectional Radon transform and the regular Radon transform has been derived in [21, 22].

Combining the inversion formula for the spherical Radon transform derived in [18] and the relation discussed in [22], we present the inversion formula for $\mathscr{R}_{P} f$ as follows.

Theorem 3. Let $f \in C^{\infty}\left(\mathbb{R}^{3}\right)$ have compact support in $B^{3}$ and be even in $x_{3}$. Then we have

$$
\begin{aligned}
& f(\mathbf{x})=-\frac{1}{64 \pi^{4}} \\
& \quad \cdot \int_{\mathcal{S}^{2}}\left(\int_{S^{1}} \int_{\mathbb{R}} \frac{\left.\left.\alpha_{3} \partial_{t} t \partial_{t} t \partial_{s} \mathscr{R}_{P} f(\boldsymbol{\theta}, s, t)\right|_{t=|\boldsymbol{\alpha}-\mathbf{x}|} \frac{\mathrm{d} s \mathrm{~d} S(\boldsymbol{\theta})}{\boldsymbol{\theta} \cdot\left(\alpha_{1}, \alpha_{2}\right)-s}\right) \mathrm{d} S(\boldsymbol{\alpha}) .}{|\boldsymbol{\alpha}-\mathbf{x}|}\right.
\end{aligned}
$$

Proof. From [21, 22], we know that, for $\boldsymbol{\theta}=\left(\theta_{1}, \theta_{2}\right) \in S^{1}$,

$$
\begin{aligned}
Q \phi(\boldsymbol{\theta}, s, t) & =\int_{\alpha_{1} \theta_{1}+\alpha_{2} \theta_{2}=s} \phi(\boldsymbol{\alpha}, t) \mathrm{d} S(\boldsymbol{\alpha}) \\
& =R\left[\Phi\left(\alpha_{1}, \alpha_{2}, t\right)\right](\boldsymbol{\theta}, s, t),
\end{aligned}
$$

where $\Phi\left(\alpha_{1}, \alpha_{2}, t\right)=2 \phi(\boldsymbol{\alpha}, t) / \alpha_{3}$ and $R$ is the $2 \mathrm{D}$ regular Radon transform of $\Phi$ with respect to the first two variables; that is,

$$
\begin{array}{r}
R\left[\Phi\left(\alpha_{1}, \alpha_{2}, t\right)\right](\boldsymbol{\omega}, s, t)=\int_{\mathbb{R}} \Phi\left(s \boldsymbol{\omega}+\nu \boldsymbol{\omega}^{\perp}, t\right) \mathrm{d} \nu, \\
\quad \text { for }(\boldsymbol{\omega}, s) \in S^{1} \times \mathbb{R} .
\end{array}
$$

Applying the inversion formula for the 2D regular Radon transform, we have

$$
\begin{aligned}
\phi(\boldsymbol{\alpha}, t) & =2^{-1} \alpha_{3} \Phi\left(\alpha_{1}, \alpha_{2}, t\right) \\
& =\frac{\alpha_{3}}{8 \pi^{2}} \int_{S^{1}} \int_{\mathbb{R}} \frac{\partial_{s} Q \phi(\boldsymbol{\omega}, s, t)}{\boldsymbol{\theta} \cdot\left(\alpha_{1}, \alpha_{2}\right)-s} \mathrm{~d} s \mathrm{~d} S(\boldsymbol{\theta}) .
\end{aligned}
$$

Also, it is well known (see, e.g., [18]) that

$$
f(\mathbf{x})=-\left.\frac{1}{8 \pi^{2}} \int_{S^{2}} \frac{\partial_{t} t \partial_{t} t R_{S} f(\boldsymbol{\alpha}, t)}{t}\right|_{t=|\boldsymbol{\alpha}-\mathbf{x}|} \mathrm{d} S(\boldsymbol{\alpha}) .
$$

Combining (12) and (13) completes the proof.

Combining Theorem 3 and (6) shows how to recover $f$ from $P$.

Corollary 4. Let $f \in C^{\infty}\left(\mathbb{R}^{3}\right)$ have compact support in $B^{3}$ and be even in $x_{3}$. Then we have

$$
\begin{aligned}
& f(\mathbf{x})=-\frac{1}{64 \pi^{4}} \\
& \quad \cdot \int_{S^{2}}\left(\int_{S^{1}} \int_{\mathbb{R}} \frac{\left.\left.\alpha_{3} \partial_{t} t \partial_{s} P(\boldsymbol{\theta}, s, t)\right|_{t=|\boldsymbol{\alpha}-\mathbf{x}|} \frac{\mathrm{d} s \mathrm{~d} S(\boldsymbol{\theta})}{\boldsymbol{\theta} \cdot\left(\alpha_{1}, \alpha_{2}\right)-s}\right) \mathrm{d} S(\boldsymbol{\alpha}) .}{|\boldsymbol{\alpha}-\mathbf{x}|}\right.
\end{aligned}
$$

\section{Conclusion}

Here we studied a Radon-type transform arising in PAT with circular detectors. We show that this transform can be decomposed into the spherical Radon transform $R_{S}$ centered at $S^{2}$ and the sectional Radon transform Q. To recover $f$ from $\mathscr{R}_{P} f$, we reconstruct $R_{S} f$ first. The formula to recover $R_{S} f$ from $R_{P} f$ is essentially (12) which is equivalent to the reconstruction of 2D Radon transform. This formula is well studied, and Matlab has "iradon" as a built-in function. The second part about the reconstruction $f$ of $R_{S} f$ is also well studied in [18-20]. Some works present numerical simulations for the inversion formula. Therefore, the numerical simulations are not within the scope of this work.

\section{Data Availability}

The data used to support the findings of this study are available from the author upon request.

\section{Conflicts of Interest}

The author declares that there are no conflicts of interest regarding the publication of this paper.

\section{Acknowledgments}

The work of S. Moon was supported by the National Research Foundation of Korea grant funded by the Korean government (MSIP) (2015R1C1A1A01051674).

\section{References}

[1] A. K. Louis and E. T. Quinto, "Local tomographic methods in sonar," in Surveys on Solution Methods for Inverse Problems, 
D. Colton, H. W. Engl, A. K. Louis, J. R. McLaughlin, and W. Rundell, Eds., pp. 147-154, Springer Vienna, 2000.

[2] C. J. Nolan and M. Cheney, "Synthetic aperture inversion," Inverse Problems, vol. 18, no. 1, pp. 221-235, 2002.

[3] A. G. Bell, "On the production and reproduction of sound by light," American Journal of Science, vol. 20, no. 118, pp. 305-324, 1880.

[4] P. Kuchment and L. Kunyansky, "Mathematics of thermoacoustic tomography," European Journal of Applied Mathematics, vol. 19, no. 2, pp. 191-224, 2008.

[5] P. Burgholzer, J. Bauer-Marschallinger, H. Grün, M. Haltmeier, and G. Paltauf, "Temporal back-projection algorithms for photoacoustic tomography with integrating line detectors," Inverse Problems, vol. 23, no. 6, pp. S65-S80, 2007.

[6] S. Gratt, K. Passler, R. Nuster, and G. Paltauf, "Photoacoustic section imaging with an integrating cylindrical detector," Biomedical Optics Express, vol. 2, no. 11, pp. 2973-2981, 2011.

[7] M. Haltmeier, "Frequency domain reconstruction for photoand thermoacoustic tomography with line detectors," Mathematical Models and Methods in Applied Sciences, vol. 19, no. 2, pp. 283-306, 2009.

[8] M. Haltmeier, O. Scherzer, P. Burgholzer, and G. Paltauf, "Thermoacoustic computed tomography with large planar receivers," Inverse Problems, vol. 20, no. 5, pp. 1663-1673, 2004.

[9] K. Kwon and S. Moon, "Reconstruction formula for photoacoustic tomography with cylindrical detectors," Journal of Mathematical Imaging and Vision, vol. 58, no. 2, pp. 265-278, 2017.

[10] G. Zangerl and O. Scherzer, "Exact reconstruction in photoacoustic tomography with circular integrating detectors II: spherical geometry," Mathematical Methods in the Applied Sciences, vol. 33, no. 15, pp. 1771-1782, 2010.

[11] G. Zangerl, O. Scherzer, and M. Haltmeier, "Circular integrating detectors in photo and thermoacoustic tomography," Inverse Problems in Science and Engineering, vol. 17, no. 1, pp. 133-142, 2009.

[12] G. Zangerl, O. Scherzer, and M. Haltmeier, "Exact series reconstruction in photoacoustic tomography with circular integrating detectors," Communications in Mathematical Sciences, vol. 7, no. 3, pp. 665-678, 2009.

[13] X. Wang, L. Jin, J. Li, Y. Ran, and B.-O. Guan, "Microfiber interferometric acoustic transducers," Optics Express, vol. 22, no. 7, pp. 8126-8135, 2014.

[14] S. Moon, "A Radon-type transform arising in photoacoustic tomography with circular detectors," Journal of Inverse and ILLPosed Problems, vol. 24, no. 3, pp. 233-244, 2016.

[15] B. Rubin, "Generalized Minkowski-Funk transforms and small denominators on the sphere," Fractional Calculus \& Applied Analysis, vol. 3, no. 2, pp. 177-203, 2000.

[16] B. Rubin, "Notes on Radon transforms in integral geometry," Fractional Calculus \& Applied Analysis, vol. 6, no. 1, pp. 25-72, 2003.

[17] D. Finch, M. Haltmeier, and Rakesh, "Inversion of spherical means and the wave equation in even dimensions," SIAM Journal on Applied Mathematics, vol. 68, no. 2, pp. 392-412, 2007.

[18] D. Finch, S. K. Patch, and Rakesh, "Determining a function from its mean values over a family of spheres," SIAM Journal on Mathematical Analysis, vol. 35, no. 5, pp. 1213-1240, 2004.

[19] L. A. Kunyansky, "Explicit inversion formulae for the spherical mean Radon transform," Inverse Problems, vol. 23, no. 1, pp. 373383, 2007.
[20] L. A. Kunyansky, "A series solution and a fast algorithm for the inversion of the spherical mean Radon transform," Inverse Problems, vol. 23, no. 6, pp. S11-S20, 2007.

[21] S. Gindikin, J. Reeds, and L. Shepp, "Spherical tomograph and spherical integral geometry," in Tomography, Impedance Imaging, and Integral Geometry: 1993 AMS-SIAM Summer Seminar on the Mathematics of Tomography, Impedance Imaging, and Integral Geometry, June 7-18, 1993, Mount Holyoke College, Massachusetts, E. T. Quinto, M. Cheney, P. Kuchment, and American Mathematical Society, Eds., Lectures in Applied Mathematics Series, pp. 83-92, American Mathematical Societ, 1994.

[22] F. Natterer and F. Wübbeling, Mathematical Methods in Image Reconstruction, SIAM Monographs on Mathematical Modeling and Computation, SIAM, Society of Industrial and Applied Mathematics, Philadelphia, PA, USA, 2001. 


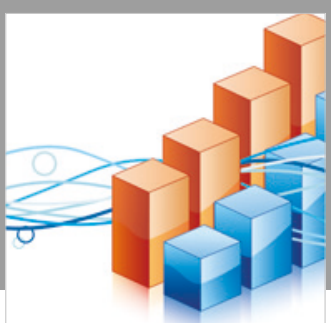

Advances in

Operations Research

\section{-n-m}
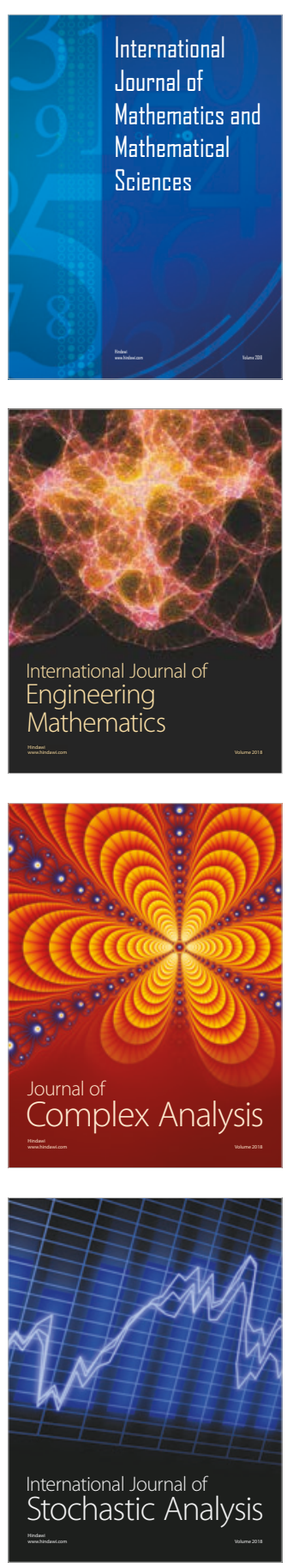
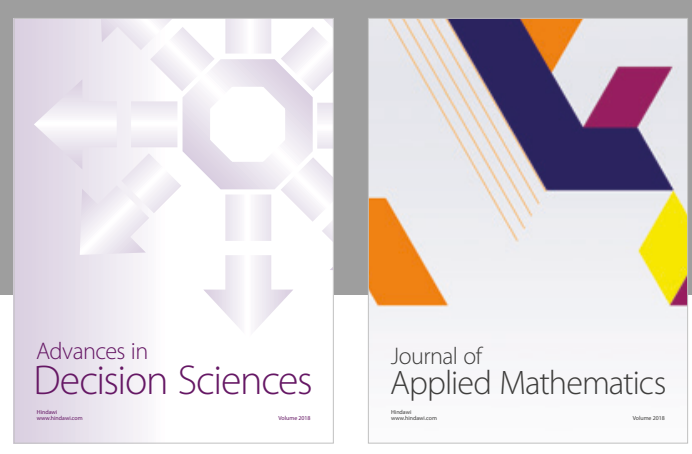

Journal of

Applied Mathematics
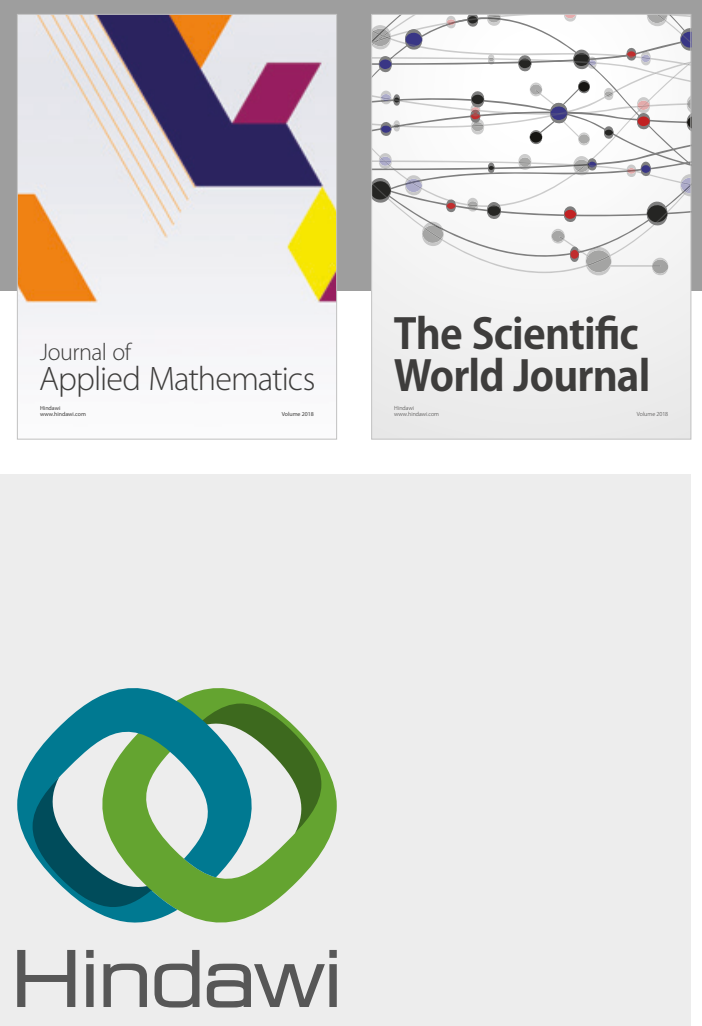

Submit your manuscripts at

www.hindawi.com

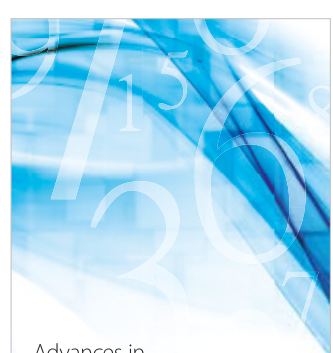

Advances in
Numerical Analysis
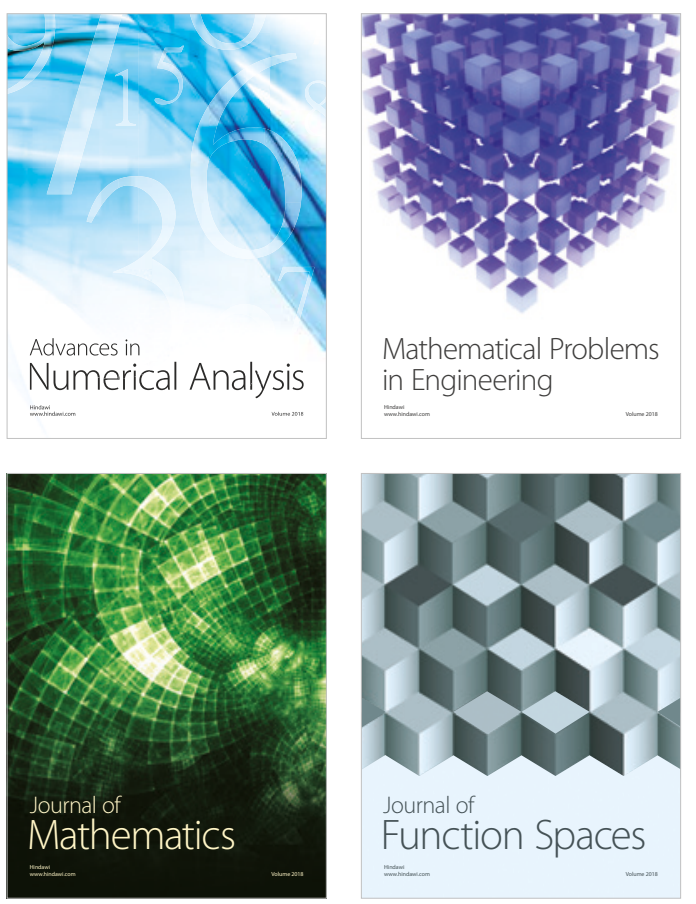

Mathematical Problems in Engineering

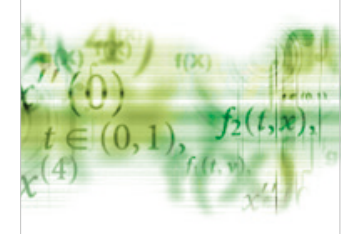

International Journal of

Differential Equations

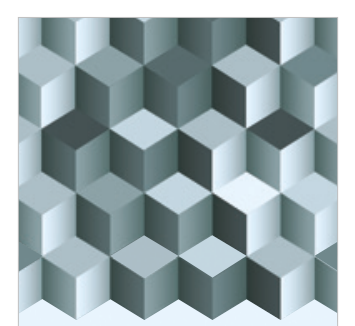

Journal of

Function Spaces

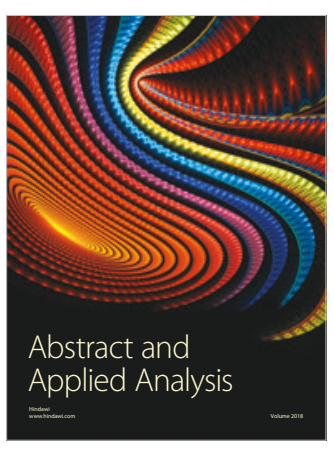

The Scientific

World Journal

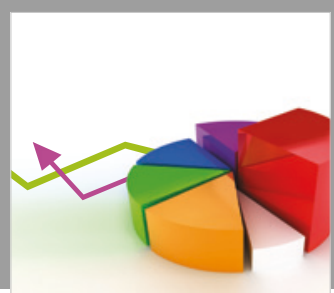

Journal of

Probability and Statistics
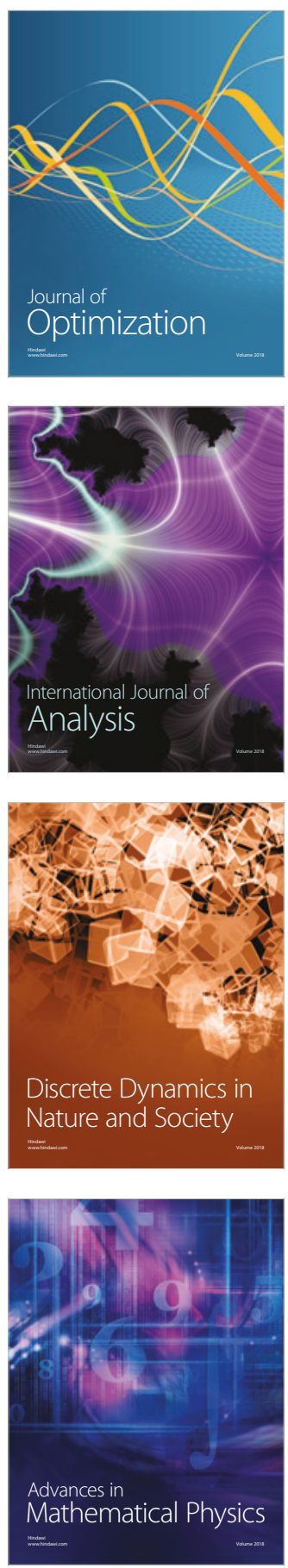